\title{
Bitcoin as an Investment and Hedge Alternative. A DCC MGARCH Model Analysis
}

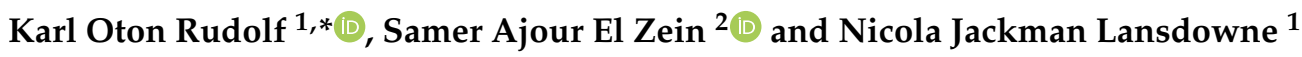 \\ 1 Research Center, Geneva Business School, Rue de, La Voie-Creuse 16, 1202 Genève, Switzerland; \\ njackman@gbsge.com \\ 2 Economics and Finance Department, EAE Business School, Calle d'Aragó, 55, 08015 Barcelona, Spain; \\ sajour@eae.es \\ * Correspondence: krudolf@gbsge.com
}

check for updates

Citation: Rudolf, Karl Oton, Samer Ajour El Zein, and Nicola Jackman Lansdowne. 2021. Bitcoin as an Investment and Hedge Alternative. A DCC MGARCH Model Analysis. Risks 9: 154. https://doi.org/ 10.3390/risks9090154

Academic Editor: Dimitrios Koutmos

Received: 7 July 2021

Accepted: 16 August 2021

Published: 26 August 2021

Publisher's Note: MDPI stays neutral with regard to jurisdictional claims in published maps and institutional affiliations.

Copyright: (c) 2021 by the authors. Licensee MDPI, Basel, Switzerland. This article is an open access article distributed under the terms and conditions of the Creative Commons Attribution (CC BY) license (https:// creativecommons.org/licenses/by/ $4.0 /)$.

\begin{abstract}
Volatility and investor sentiment have been factors for the slow adoption rate of Bitcoin (BTC) that was first recognized in 2008 as a potential store of value, investment vehicle and a hedge alternative to gold during a recession. The purpose of this applied mathematics study will use a multivariate DCC GARCH model. Bitcoin holds its ground in volatility. This study examines Bitcoin as an investment and hedge alternative to gold as well as the major stock index. To perform the research to explore the viability of Bitcoin as an investment and hedge alternative to gold, the authors conducted a DCC GARCH model analysis. The findings of this research paper confirm Bitcoin's cyclical performance between volatility and adoption. The findings give a strong ground for Bitcoin as the new digital currency, store of value, medium of exchange, and a unit of account and incentivize further research by theorists, scholars and examiners. The significance of this applied mathematics research and analysis will allow an unstoppable, incorruptible, and uncontrollable store of value, and investment vehicle, without governmental or institutional intervention. This study contributes by comparing and contrasting volatility stability based on the return levels of each Bitcoin on major indexes traded with BTC (based on fiat currencies) and gold.
\end{abstract}

Keywords: DCC MGARCH; bitcoin; blockchain; cryptocurrency; risks

\section{Introduction}

Recent notable inventive forcing functions include computers, the start of space exploration, electrification of the world, sustainability in agriculture, and improvements in materials science (Senge et al. 2008). The first taste of decentralisation technology was information through communication by internet (Datta et al. 2010). Decentralised technology is currently present in cryptocurrencies (Scott et al. 2017) and has the potential to be explored much more. Modelling investor perception is straightforward, but modelling the impact of halving and volatility is complicated (Kurtz and Schrank 2007). Volatility and adoption is a 'chicken and egg' concept with Bitcoin (BTC). Bitcoin is volatile because it does not have mass adoption. Bitcoin as an investment and hedge alternative to gold has been tackled in the literature and this research will explore its application based on a dynamic conditional correlation (DCC) generalized autoregressive conditional heteroskedasticity (GARCH) model analysis. When investors are making long-term decisions, Bitcoin becomes less speculation and more of a medium of exchange (Yermack 2015) at which point, volatility should drop rapidly as volatility acts as a symptom of size in BTC (the cyclical argument). In order for BTC to reach full adoption, there have to be motivations that override the initial difficulty of dealing with volatility. The more volume, the greater the liquidity, and this will have an immediate impact on volatility (Frino et al. 2011). Modelling investor perception is straightforward, yet modelling the impact of halving, and volatility is complicated (Garman and Klass 1980). Those motivations already exist in many parts of the world which is why this study provides utility. The paper is organised into five 
major sections. First the introduction, followed by the literature review of previous work discussing Bitcoin's potential ramifications, the methodology, experimental results analysis, and a conclusion.

\section{Literature Review}

\subsection{Proto-Money}

Initial trade was conducted through barter, with the big disadvantage being the balance between offer and demand. With time, humans evolved towards a need to create and hold collectables such as flint, animal teeth and shells. This was the ultimate evolutionary advantage as a form of generational wealth preservation and storage. Collectables made trade possible as a form of proto money and not yet a medium of exchange (MoE). Szabo (2002) notes that collectables had very low velocity, although a heirloom added substantial value increase over time. Similarly, as in the global financial crisis of 2008, Rome in 33AD experienced a liquidity crisis and government bailout through the treasury banks (Elliott 2015).

Money is an invention by traders, IOUs raised by merchants and banks as well as government bonds by kings to finance war. Achieving Nash equilibrium, or the feedback loop which drives societies to converge on a single store of value (SoV), facilitates trade, the division of labor, and paves the way for the creation of a civilization (Boyapati 2018). As trading routes developed, so did competition for stores of value. Merchants would benefit from foreign SoVs to facilitate continuous trade. The 19th century was the first-time humanity converged on one SoV-gold, followed by the first flow of trade worldwide. Subsequent monetary systems were created, such as the Bretton Woods system (Knafo 2006). Gold became a SoV and a treasury reserve asset due to eight factors: durability, portability, fungibility, verifiability, divisibility, scarcity (or lack of human ingenuity to make more), established history and censorship resistance, such as India's Gold Control Act of 1968.

The 20th-century issuance of money (fiat currency) has been controlled by governments, creating a belief that money is merely a MoE, and not a SoV. Historically, gold went through "The Four Pillars". The first, serving as a collectible, then as a store of value with an eventual plateau of demand, later as a medium of exchange due to purchasing power stabilization, and finally as a unit of account for denominating payments. Today, gold is a monetary good that is partly monetized due to the fact that it is being treated as a SoV/treasury reserve asset by central banks. Various currencies act with their own combination of the Four Pillars, e.g., the dollar (USD) as a unit, MoE, and SoV, as opposed to inflation prominent currencies such as the Argentine peso, which is only an MoE. Currently, Bitcoin still does not act as a MoE, merely as a digital SoV. Its inherent finite supply and deflationary nature make it historically highly speculative, thus volatility represents its biggest obstacle as a hedge alternative to gold, major indexes as a SoV and fiat money as a form of payment. Once volatility stabilization is achieved, mass adoption will follow (Breedlove 2019).

\subsection{Bitcoin}

A consequence of the 2008 financial crisis was the creation of the first engineered monetary network. First mentioned in November 2008, a paper was published under the title "A Peer to Peer Electronic Cash System" under the pseudonym 'Satoshi Nakamoto' (Nakamoto 2009). In computer science, (Lamport et al. 2019) solved the Byzantine Generals Problem, however, this was achieved with the assumption of semi-known identities. 'Satoshi' effectively created crypto-economics, with the concept of using economic resources to limit the number of participating identities through proof of work in the blockchain network.

The Bitcoin tokens (BTC) created were developed to be a manifestation of collectibles, a store of value, a medium of exchange, and a unit of account, which can be transmitted through a decentralized peer to peer network in time and space through the blockchain 
database vacuum. The total supply of memetic Bitcoin stands at 21 million. Every four years, the number of Bitcoins mined halves, ending full production by 2140.

Without any governmental guarantee, Bitcoin's future as a store value is questionable. Bitcoin tokens cannot be valued via discounted cash flow models such as in inflation predisposed securities and/or asset classes. Bitcoin belongs to its unique asset class and stands to be valued through other means. One such means is that of Metcalfe's Law (M.L.). The law states that the value or utility of a network is proportional to the number of users of the network, in other words, that utility is a square function (utility $=n \times 2$ ) (Peterson 2018). In network effects, users become the biggest champions of the network, thus the first mover network stands to innovate the fastest and survive the longest. This would make BTC the potential reserve currency or asset of the monetary network. BTC seemed to be historically obeying Metcalfe's Law between 2010 and 2018.

\subsection{Adoption History}

Bitcoin started gaining popularity in 2011. Wikileaks started accepting Bitcoin as donations (Grinberg 2011). Between 2011 and 2012, several articles and conferences were held about Bitcoin, and financial scandals occurred (Takemoto and Knight 2014); moreover, online exchanges as well as wallets were created for cryptocurrencies (Stross 2012), which sparked interest. However, Bitcoin did not receive mass recognition until 2013. The 2013 Cypriot Financial Crisis led the government to accept a bailout by the EU and the IMF and funded it by imposing a bank levy on all deposits (Luther and Salter 2017). It should be noted that Bitcoin's demand was also driven by illegal activities on darknet markets such as Silk Road, due to its anonymity factor (Tsertsvadze and Khurtsia 2015). Several retailers began accepting Bitcoin (Acharya and Dunn 2014), and in 2014 the IRS classified Bitcoin an asset and not a currency. The 2020 pandemic, and halving, demonstrated further mass adoption and lower volatility compared to historical halvings as predicted (Masters 2019).

\subsection{Austrian Economics}

In 2012, the European Central Bank (ECB) published a study, mentioning that Bitcoin's roots can be found in Austrian economics (Nabilou 2020). One of the foundations of Austrian economics is the business cycle theory, whereby Freidrich Hayek and Ludwig von Mises claim that cycles are not a natural phenomenon but are primarily driven by central bank intervention and fractional reserve banking. The reason being that the government sends false signals to production segments of the economy by lowering interest rates below the free market rate. This in turn causes overconsumption and malinvestments, causing cycles. The aforementioned economists connected their theory with the 'Regression Theorem' through the Gold Standard (Davidson and Block 2015). As the Gold Standard fell, fiat arose. The Austrian school of thought states that retention of fiat currency value can be done if governments monopolise its issuance (Clegg 2014). Hayek believed that the only way to ensure the prevention of currency inflation was by letting market participants choose multiple currencies within their own country (Hayek 1976). Bitcoin obeys the Austrian definition of currency as Hayek proposed as it serves as a MoE (medium of exchange).

\subsection{Keynesian Economics}

Keynesian models are used by central banks to determine interest rates, assuming business cycles are caused by aggregate demand (AG) (Lavoie 2014). Keynesian theory describes the inverse relationship between inflation and unemployment through the Phillips curve. The theory suggests fluctuations are decreased by monetary and fiscal policy when the unemployment rate goes above the natural employment rate. Keynes believed participants value money as a store of value and a form of transaction due to liquidity preferences. The demand for money would depend on current interest rates due to precautionary, speculative and transactional motives of participants. Since 2013, to promote increasing adoption, and technological innovation and volatility stabilisation, corporations such as 
Blockstream have improved infrastructure to promote scalability and create stability for the decentralised network. A 2014 Goldman Sachs paper estimated $\mathrm{a} \cong 1 \%$ cost for sending payments globally with BTC, as opposed to a $\cong 7.7 \%$ through traditional methods.

\subsection{Electricity Usage and Scaling}

For transaction time purposes, Bitcoin's biggest obstacle at becoming a unit of account is again its volatility, as it is not on par with fiat's yet and lack of correlation information compared to traditional assets. Currently, merchants use middlemen and convert BTC to fiat immediately. Previous debates have concerned Bitcoin's electricity usage due to demanding cryptographic calculations by miners to verify transactions. The hashing costs are as of now subsidised by BTC rewards. As the rewards get smaller, so will consumption. Energy consumption has been a controversial topic in crypto mining. Estimates vary considerably from study to study, as not all studies use both metrics: minimum energy consumption and the profitable maximum. Küfeoglu and Özkuran (2019) mention that the choice of hardware is crucial to miners, and that electricity costs are impossible to predict due to unforeseeable electrical innovations, hardware choices and BTCs direct impact on mining thus consumption. It's important to note that hardware always beats software.

Previous papers mentioned that the weakness with scaling was quadratic validation. This is the attack vector that makes scalability difficult, as one cannot have decentralisation and scalability on the same chain. The authors note that this is not achieved by pushing more transactions through a system with a data limit. Instead, scalability is achieved by mimicking other settlement payment networks which is by introducing deferred settlement on various layers. For fast transactions the Lightning Network suffices, but more importantly, the deployment of satellites can ensure APIs to broadcast larger files, as well as the introduction of various chains can help with scalability. The authors propose distinct features for chains.

These distinct features include a unique chain for traders and a faster settlement speed, more privacy for the main chain, one for bigger block enthusiasts, and rollups. Each chain could have its own fee structure to cover costs for the miners. This would keep decentralisation the main theme.

Cryptography is the building block for the digital world and Bitcoin addresses the big gap in privacy for financial dealings as well as wealth storage. Encryption was created for pgp emails, chat and voice communications, but not electronic money outside of government (Borradaile 2021). Countries that have banned encryption have the worst human rights records (Fukami et al. 2021). This makes it clear that what society in the current civilisation expects is the right to use encryption (Stevens and Allen-Robertson 2021). Bitcoin's decentralisation brings the benefit of full public user account auditability (unlike confidentiality of addresses and value transactions in other cryptocurrencies), albeit with the cost of full absolute privacy. Bitcoin does in fact use understood and dependable building blocks such as elliptic curve cryptography, hashing, symmetric ciphers and so forth. In addition, Bitcoin has received incremental improvements over time such as lightning wallets, the Schnorr signature and taproot which reduce fingerprinting, and give the prospects in harmonising many improvements.

\subsection{Bitcoin and Traditional Assets as a Hedge Tool}

Research in cryptocurrencies has continued to consider whether Bitcoin possesses a safe haven property in comparison with traditional assets (gold and US equities) (Selmi et al. 2018) as well the hedging ability of Bitcoin against other assets (Umar et al. 2021). Despite the ongoing debate on the usefulness of Bitcoin for investments (Bouri et al. 2017), it acts as a diversifier for commodities (Selmi et al. 2018), for currencies (Regaieg et al. 2020), and as a hedge tool for most investors (Stensås et al. 2019). Shahzad et al. (2019) consider Bitcoin a better safe haven investment than gold and commodities, whereas Smales (2019) considers it more volatile even in normal times with a downside risk spillover to traditional assets and should be monitored carefully for the sake of financial stability (Zhang et al. 2021). Bitcoin 
gold is a safe haven asset for global crude oil markets (Dutta et al. 2020). Bitcoin, gold, and the commodity index can be considered weak safe-haven assets in some cases depending on the market and economic conditions in the countries (Kliber et al. 2019). Moreover, Bitcoin, gold and VIX (Chicago Board Options Exchange's CBOE Volatility Index) futures have a time-varying hedging role in some BRICS countries (Brazil, Russia, India, China, and South Africa), with gold offering a more diversification benefits (Shahzad et al. 2019). Nevertheless, USD-pegged stablecoins perform better than gold-pegged stablecoins in extreme risk reductions (Wang et al. 2020). Thus, both gold and Bitcoin have attractive features of risk management and portfolio risk (Hossain et al. 2020) and their role in the market is yet to be revisited (Jiang et al. 2021).

\section{COVID19 and the Future}

\subsection{Stores of Value}

Traditional stores of value are valuable due to their scarcity and if large networks of people want them (gold, land, web addresses in cyberspace, trademarks, art, well managed Fiat), or due to their ability to generate income (companies, real estate, bonds, loans, employees, taxpayers). They have utility but will never be a SoV as they are not fully scarce. Scarce things in the world have conservation energy such as in thermodynamics.

When conservative engineering is removed, boats do not float, airplanes do not fly, pneumatic and hydraulic systems do not work, electrical engineering systems fail, and software cannot function. Moreover, BTC's true scarcity stems from two dynamics. The first order dynamic is a decentralised network running on thousands of nodes, and no one node can change the consensus, thus it is impossible to change the network's operation without taking over the whole network (which is decentralized with massive inertia). The second order dynamic is cloning. Cloning of Bitcoin has failed thousands of times due to BTCs multiple network effects (Maddox and Heemsbergen 2021).

\subsection{Debt, Modern Monetary Theory and Bitcoin}

Modern monetary theory is described as a heterodox macroeconomic framework that supports currency as a public monopoly and unemployment as evidence that the currency issuers are over restricting the supply of financial assets needed to pay taxes and satisfy saving desires (Wray 2015). Connors and Mitchell (2017) simply claim the MMT is the current establishment that the world has lived in since 1971, that currency has no intrinsic value and governments are in fact not constrained in their spending by a need to raise revenue, leading to high debt to GDP ratios around the world. Classical Austrian economists believe that this stimulus of fiscal policy will lead to heavy long-term risks as at the start of the century, the equation for catastrophic societal consequences was set by unconstrained governmental spending, loose central bank policies and money printing. Bitcoin is the antithesis to MMT's thesis, although they are united in their pursuit of evolving the conversation beyond economic theories camps. 'Satoshi' once wrote that the root of problems of fiat currencies is the trust required to make them function. However, history is laden with central banks that continuously break that trust to not debase their currency, thus devaluating assets. As the spectre of inflation increases, money stored in banks will struggle to keep pace, money invested in assets such as securities and real estate will keep pace, and money stored in gold and Bitcoin will outrun scourge. At the time of finalising this research project $(1 / 13 / 2021)$, the market capitalisation of Bitcoin stood at $\$ 629$ billion, and gold was approximately $\$ 10$ trillion (Fröhlich et al. 2021). Using Bitcoin as the digital gold metric, Bitcoin was undervalued by a multiple of 16, establishing itself at roughly $\$ 500,000$ per coin. However, Bitcoin is not seen as displacing fiat currencies, but merely a currency alternative, an asset alternative and a hedge for foreign exchanges held by governments. The hope for currencies is that central banks start diversifying their fiat holdings party into Bitcoin, pushing a BTC upwards of the $\$ 600,000$ range. The focus of this research paper and study is to determine if Bitcoin can serve as a return asset alternative to the major stock index, a medium of exchange, a store of value and a hedge alternative to 
gold. If this occurs, Bitcoin can be seen as a unit of account, or reserve currency, for banks in cyberspace. Economic feasibility will be evaluated without the internal factors of concerns labeled by economists. As previously mentioned, volatility, price stabilisation, lack of correlation knowledge, and lack of forecasting are the biggest obstacles for such adoption. BTC so far seems to be functional without governmental oversight while operating in a legally grey area. Traditional volatility models measuring such as moving average deviation or standard deviation do not consider that the variance covariance of returns can be volatile during downturns and crises. The said methods account for constant volatility, unlike the generalized autoregressive conditional heteroskedasticity (GARCH) models which consider time-varying volatility which of greater importance in financial modelling. Process models such as the autoregressive moving average (ARMA) are used to model the conditional expectation of a process given its past, but the conditional variance is constant i.e., it cannot capture non-constant volatility. Thus, a GARCH model would be most appropriate for an asset like BTC due to its nature and the 2020 post-halving adoption wave.

Historically, Gronwald (2014) used a GARCH model to conclude BTC was still an immature market due to BTC prices being too dependent on speculation. Dyhrberg (2016a) used asymmetric GARCH models to determine that BTC was useful as a hedge for risk management against stock indexes. Lastly, Bouri et al. (2017) noted that BTC might not actually serve as a hedge instrument, but as an effective diversifier using forecasted GARCH volatility models. Moreover, to better understand the role of cryptocurrencies as a store value, recent studies (Catania et al. 2019) highlighted the forecasting performance of cryptocurrencies by vector autoregressive model with and without time-varying volatility (Bohte and Rossini 2019). Lastly, Bianchi et al. (2020) investigated the relationship between the returns on stable-coins and major cryptocurrency pairs within the context of a large vector auto-regressive (VAR) model, concluding that stable coins such as Tether do correlate with lagged cryptocurrency returns. This justifies cryptocurrency markets as a role of alternative investments.

\subsection{United States Dollar (USD), Gold and the Future}

The collapse of the Bretton Woods system and termination of the peg to Gold in 1971 rendered the USD a fiat currency. Since then, the USD has been seen as the global reserve currency, dominating trade, and till now, a reliable store of value due to good management and governance by the Federal Reserve, resilience and strength of the U.S. economy.

The pre-pandemic consensus was a debt leveraged economy in all sectors. COVID19 took direct monetisation of massive fiscal spending from theory to practice at an unprecedented pace. Further devaluation of the dollar is poised to occur as the Treasury has been running a budget deficit every year since 2009, forcing the Fed's balance sheet to grow between $\$ 8.5$ and $\$ 10$ trillion in 2021 (unambiguous debt monetization) (Putnam 2020). Instead of running a budget surplus during the pro-business cycle for the past decade and keeping cash on hand, the government decided to adopt Friedman's "helicopter money" as cash to combat the 2020 downturn.

COVID-19 pushed the USD-debt ratio towards 136\% at the end of 2020 (Amadeo and Boyle 2020), forcing investors to seek refuge in instruments that respond fastest to increases in money supply. What is apparent is that economic downturns are primarily fueled by a global ramp up of debt in modern monetary theory (MMT). Jones and Giorgianni (2020) estimate the economy's worldwide debt ratio may increase by $50 \%$ of GDP over the next year and a half. Concerns over the USD arise if M2 growth exceeds real output growth in the next demi-decade, if a big monetary overhang in the ramp up will fuel consumer price inflation or not.

Stimulating the economy with debt and lowering interest rates would no longer be effective and Keynesian policies have become somewhat haggard. Governments deal with debt in three forms: a hard default, austerity, and a soft default. Since the USD is the reserve currency, a hard default would have catastrophic effects worldwide, thus it is 
hard to assume this would be the solution. Austerity measures, as previously mentioned, would be difficult to implement due to the increased social unrest generated around the implementation of austerity measures, (Ponticelli and Voth 2011), and tax increases with spending cuts, which leads to decreasing GDP, further aggravating the debt. A soft default is most probable and suitable for governments to deleverage, although it would mean devaluing the USD to erode real value.

It is worth noting that the pre-COVID inflation may have exhibited itself via. neoliberal policies which favour capital over labour. In the 18th century, Richard Cantillon noticed that when economic capital injection occurs, its outcomes were not evenly distributed (Cantillon effect). Participants who first received the money would spend it on goods and services, and consequently increasing price, which the majority would have to endure until the capital reached everyone (if ever). During this period, inflation would be evenly distributed, unlike purchasing power (Rosales 2021). In that time, early participants would only benefit due to their proximity to the king or new gold deposits. This occurs similarly in the current monetary system and raises concerns.

\section{Data and Methodology}

\subsection{Data}

There is no consensus in previous literature about which factors affect the exchange rate of fiat currencies with BTC (De Grauwe 1988) but it is generally accepted as the standard reason for macroeconomic variables (inflation, money supply, interest rates, exports, GDP) (Antonakakis and Darby 2013). Since BTC is decentralised, it could potentially be considered an international currency (Andersen and Bollerslev 1988). In order to determine the most appropriate variables to compare, one must consider the currency pairs, or countries with which BTC is most actively traded (Engel and West 2005). Data are collected from Coinhills which provides a list of the most traded fiat currencies for trading Bitcoin from over 11,500 markets and 120 exchanges registered with Coinhills (Kim 2021). One can infer that, as expected, the US dollar dominates trade volume as it is the world reserve currency, the Japanese yen in second place as it has gained a lead over the Chinese Yuan, since the Chinese government ban on crypto currency exchanges in September 2017 (Cuñado et al. 2020) followed by the Korean won due to local governmental adoption and regulation, and lastly the euro. Consequently, it can be assumed that the majority of volatility and correlation arises from the United States, Japan, South Korea and the European Union. Previous studies examined the top currency pairs with Bitcoin (Dyhrberg 2016a), as well as with gold and macroeconomic indicators such as $10 \mathrm{Y}$ government bond yields, top indexes, three-month LIBOR rates. Cermak (2017) concluded that BTC does not yet satisfy the criteria of a currency, either $\mathrm{MoE}$ or a SoV, and that macroeconomic explanatory variables are significant measures of forecasted volatility and return. The authors of this research will be comparing Bitcoin as an investment vehicle to the biggest index of the dominant currency, the S\&P 500, and gold with Bitcoin as digital gold, since they share similarities (barter, SoV, MoE etc.). The study will be conducted in the form of three assets. These are: the SPDR Gold Trust ETF (ticker: GLD) representing gold, the Vanguard 500 Index Fund (ticker: VOO) representing the S\&P 500, and the Grayscale Bitcoin Trust Fund (ticker: GBTC) representing Bitcoin. Both ETFs and the Trust track their respective asset, or commodity. The primary reason for an ETF and Trust-based study is that the source proving data, Yahoo Finance, is missing historical data about the BTC-USD pair and the S\&P 500, at Bitcoin's halving event in May 2020, disallowing examination. Secondary reasons are that Greyscale holds 572,644 BTC after purchasing 21\% of newly mined Bitcoin in April 2020, is an SEC registered asset, and shares are OTC traded which makes them applicable for tax advantaged accounts and available both for institutional and retail investors (including retirement accounts) through standard brokers. Moreover, electric wallets have not fully matured yet, and instead, Greyscale (GBTC) is both a financial vehicle and hedge alternative that enables investors of all ages to trade shares in trusts holding many BTC. 
The reason for excluding currency pair comparisons is that previous studies have already examined these using the said methodology.

Furthermore, the monetary system economy is inherently debt-leveraged, and the ability of currency appreciation stems from a country's ability to favourably manage trade, which is rarely the case to justify comparing to currencies as a SoV or a certain MoE. The data range between 29 January 2018 and 29 January 2021, and not prior to 2018, as previous volatility modelling research was conducted for the 2017 bull bubble. For clarification, a bull run bubble is defined by when a bull market occurs for a prolonged period of time, where prices rise much higher than the value of the underlying asset. The economic theory of reflexivity further explains bubbles via. market sentiment, economic fundamentals, feedback loops connected to them and challenges the concepts of economic equilibrium, rational expectations as well as the efficient market hypothesis (Griffin and Shams 2020). The 2017 BTC bubble was primarily driven by unregulated market manipulation via Chinese exchanges (Chen et al. 2019). All modelling will be done with the programming language r. All data are collected from Yahoo Finance. One limitation identified by the authors is that explanatory variables might be missing observations as markets are closed on weekends and bank holidays, consequently the researcher will use a model with a foundation of the multivariate GARCH process (Poon 2005), as has been proved to be the most appropriate model for stock index, commodity (Hansen and Lunde 2005), and currencies (Brownlees et al. 2011). The said models are not only used in modeling historical performance but in forecasting multi-period volatility, correlation, within others.

\subsection{Models Autoregressive Conditional Heteroskedasticity (ARCH) and Generalized Autoregressive Conditional Heteroskedasticity (GARCH)}

In order to understand the theory behind GARCH models, one must understand the ARCH model first built by Robert Engle (1982) as a mechanism of estimating time varying volatility. Moreover, the univariate GARCH is the most commonly accepted and robust model for estimating and forecasting volatility of returns of an asset.

\subsection{Model Used: Dynamic Conditional Correlation (DCC)-GARCH and $r$}

For this experiment the researcher will be using an evolved model of the GARCH, a multivariate GARCH model (MGARCH), which allows the covariance matrix of the dependent variables conditional on past history (Bauwens et al. 2006) to follow a flexible dynamic structure (Laurent et al. 2012). The importance of all multivariate GARCH models is that they stem from the fact that the current volatility of one time series is not only influenced by its own past innovation (such as in the GARCH model), but also by past innovations to volatilities of other time series which make the best model for forecasting the aforementioned assets and compare them. Andersson-Säll and Lindskog (2019) note that the MGARCH model with the best BIC (Schwarz's Bayesian Information Criterion) score is the DCC-GARCH model (Dynamic Conditional Correlation). The authors will model the volatility of a vector of asset classes (GLD, GBTC, VOO), using the 'rmgarch', 'quantmod' and 'rugarch' packages (in r). These packages will be applied to estimate a multivariate volatility model for the returns of mentioned asset classes, sticking with a dynamic conditional correlation (DCC) method. There are four common methods for MGARCH models, which trade off between flexibility and parsimony. In $\mathrm{r}$, the DCC method estimates the individual GARCH type models (i.e., individual assets). These are then used to standardise individual residuals. In the second step, the authors will specify the correlation dynamics of these standardised residuals. This model is useful in econometrics and management understanding in predicting dependence in the comovement of asset returns. As an example, asset pricing depends on the covariance of the assets in a diversified portfolio. Orskaug (2009) notes that financial volatilities, over time, move together more or less closely across asset classes and MGARCH models provide better insight into asset pricing, portfolio selection, hedging and value at risk forecasts. 
Orskaug (2009) describes the DCC-GARCH model in Equation (1).

$$
r_{t}=\mu_{t}+a_{t} \alpha_{t}=H_{t}^{1 / 2} Z_{t} H_{t}=D_{t} R_{t} D_{t}
$$

where:

$r_{t}=n \times 1$ vector log returns of $\mathrm{n}$ assets at time $t$.

$\alpha_{t}=n \times 1$ vector of mean corrected returns of $\mathrm{n}$ assets at time $t$, i.e., $\mathrm{E}\left[\alpha_{t}\right]=0$.

$\mu_{t}=n \times 1$ vector of the expected value of the conditional $r_{t}$.

$H_{t}=n \times n$ matrix of conditional variances of $\alpha_{t}$ at time $t$.

$H_{t}^{1 / 2}=$ any $n \times n$ matrix at time $t$ such that $H_{t}$ is the conditional variance matrix of $\alpha_{t}$.

$H_{t}^{1 / 2}$ may be obtained by a Cholesky factorization of $H_{t}$.

$D_{t}=n \times n$ diagonal matrix of conditional standard deviations of $\alpha_{t}$ at time $t$.

$R_{t}=n \times n$ conditional correlation matrix of $\alpha_{t}$ at time $t$.

$Z_{t}=n \times 1$ vector of iid errors such that $\mathrm{E}\left[Z_{t}\right]=0$ and $\mathrm{E}\left[Z_{t} Z_{t}^{T}\right]=\mathrm{I}$.

The elements in the diagonal matrix $D_{t}$ are standard deviations from univariate models.

DCC - GARCH:

$$
D_{t}=\left[\begin{array}{cccc}
\sqrt{h_{1 t}} & 0 & \cdots & 0 \\
0 & \sqrt{h_{2 t}} & \ddots & \vdots \\
\vdots & \ddots & \ddots & 0 \\
0 & \cdots & 0 & \sqrt{h_{n t}}
\end{array}\right]
$$

where:

$$
h_{i t}=\alpha_{i 0}+\sum_{q=1}^{Q 1} \alpha_{i q} \alpha_{i, t-q}^{2}+\sum_{p=1}^{P i} \beta_{i p} h_{i, t}-p
$$

$R_{t}$ is the conditional correlation matrix of the standardized disturbances $t$, i.e.,

$$
\epsilon_{t}=D_{t}^{-1} \alpha_{t} \sim N\left(0, R_{t}\right)
$$

Since $R_{t}$ is a correlation matrix it is symmetric.

$$
R_{t}=\left[\begin{array}{ccccc}
1 & p 12, t & p 13, t & \ldots & p 1 n, t \\
p 12, t & 1 & p 23, t & \cdots & p 2 n, t \\
p 13, t & p 23, t & 1 & \ddots & \vdots \\
\vdots & \vdots & \ddots & \ddots & p n-1, n, t \\
p 1 n, t & 2 n, t & \cdots & p n-1, n, t & 1
\end{array}\right]
$$

The elements of $H_{t}=D_{t} R_{t} D_{t}$ is:

$$
\left[H_{t}\right]=\sqrt{h_{i t} h_{j t} p_{i j}}
$$

where: $p_{i j}=1$.

Orskaug (2009) notes that there are different forms of $R_{t}$. Two requirements need to be considered:

(1) $H_{t}$ has to be positive definite as it is a covariance matrix. To ensure $H_{t}$ will be a positive definite, $R_{t}$ has to be positive definite ( $D_{t}$ is positive definite since all the diagonal elements are positive).

(2) All the elements in the correlation matrix $R_{t}$ have to be equal or less than 1 by definition.

To ensure both of these requirements in the DCC GARCH model, $R_{t}$ is decomposed into Equation:

$$
R_{t}=Q_{t}^{*-1} Q_{t} Q_{t}^{*-1}
$$




$$
Q_{t}=(1-a-b) \bar{Q}+\alpha \epsilon_{t-1} \epsilon_{t-1}^{T}+b Q_{t-1}
$$

where $\bar{Q}=\operatorname{Cov}\left[\epsilon_{t} \epsilon_{t}^{T}\right]=\mathrm{E}\left[\epsilon_{t} \epsilon_{t}^{T}\right]$ is the unconditional covariance matrix of the standardized errors $t . \bar{Q}$ can be estimated as:

$$
\bar{Q}=\frac{1}{T} \sum_{t=1}^{T} \epsilon_{t} \epsilon_{t}^{T}
$$

The parameters a and $\mathrm{b}$ are scalars, and $Q_{t}^{*}$ is a diagonal matrix with the square root of the diagonal elements of $Q_{t}$ at the diagonal:

$$
Q_{t}=\left[\begin{array}{cccc}
\sqrt{q 11 t} & 0 & \cdots & 0 \\
0 & \sqrt{q 22 t} & \ddots & \vdots \\
\vdots & \ddots & \ddots & 0 \\
0 & \cdots & 0 & \sqrt{q n n t}
\end{array}\right]
$$

$Q_{t}^{*}$ rescales the elements in $Q_{t}$ to ensure the second requirement using;

$$
\left|p_{i j}\right|=\left|\frac{q_{i j t}}{\sqrt{q_{i i t} q_{i i t}}}\right| \leq 1 \text {. }
$$

Moreover, $Q_{t}$ to be positive definite to ensure $R_{t}$ to be positive definite. There are parameters $\mathrm{a}$ and $\mathrm{b}$ to guarantee $H_{t}$ to be positive definite. In addition to conditions for the univariate GARCH models, to ensure positive unconditional variances, the scalars $\mathrm{a}$ and $\mathrm{b}$ must satisfy: $\mathrm{a} \geq 0, b \geq 0$ and $a+b<1$.

In addition, $Q_{0}$, the starting value of $Q_{t}$ has to be positive definite to guarantee $H_{t}$ to be positive definite.

The correlation structure can be extended to the general DCC (M, N)-GARCH model described in Equation:

$$
Q_{t}=\left(1-\sum_{m=1}^{M} \alpha_{m}-\sum_{n=1}^{N} b_{n}\right) \bar{Q}+\sum_{m=1}^{M} \alpha_{m} \alpha_{t-1} \alpha_{t-1}^{T}+\sum_{n=1}^{N} b_{n} Q_{t-1}
$$

The creator of the 'rmgarch' package, Ghalanos (2019), notes that the conditional mean dynamics of the package allow for either a constant or univariate AR or vector AR (VAR) model to be fit, and are described in the 'rugarch' package (used for a simple GARCH model). He adds that for the DCC-based models, the constant AR model is jointly estimated with the first stage GARCH dynamics, and that when using a constant in the DCC model, standard errors are calculated for all stage parameters using a partitioned standard error matrix. In the case of the VAR model (vector autoregression), the joint estimation of standard errors is not practical due to the dimensionality of the system. Engle (1982) created the DCC model in which the conditional quasi-correlations Rtfollow the GARCH process. The DCC model is notably more flexible than other MGARCH models (such as CCC), without introducing an inestimable number of parameters for a reasonable number of series. The reason for an extensive explanation is that multivariate models (any MGARCH models) are hard to estimate, and in $r$, estimating univariate models beforehand (GARCH), leads to a somewhat more robust empirical method, especially when comparing securities, and commodities from different asset classes.

\section{Expectations}

\section{Returns, Volatility, Correlation}

Bitcoin does not have any internal variables except intervention events such as halvings, governmental closure of the cyberspace (world wide web/internet), hacks, depreciation of fiat currencies, and halving periods which cannot all be examined as a function of returns. This is true as BTC's value is driven by market forces and, as of now, does not have a high intrinsic value. The returns are expected to increase and be correlated with post-recession or worldwide index crashes, as well as lower volatility after each halving. 
A small crash occurs after every bull run, and runs are expected to go for a long time. GLD (Gold) and VOO (Vanguard 500 Index Fund) return percentage is expected to be an inverse relationship due to their nature with a strong correlation and similar volatility levels. Moreover, previous days' volatility will be a significant indicator in explaining today's volatility (Takaishi 2020). More importantly, a good predictor for Bitcoin as BTC, till now has been traded based on speculation and halvings. Due to BTC being traded the most with the USD, the impact of the S\&P 500, or VOO is expected to be a strong variable in Bitcoin's performance. GLD and VOO are expected to once more have a strong inverse relationship. GBTC and GLD are expected to perform somewhat similarly in this examination since regulations have severely tightened and matured since 2017. This is to say that the authors believe similar results will be shown in terms of volatility and correlation, due to the post 2017 adoption wave, but more importantly higher returns of Bitcoin as its digital nature allows a faster reaction velocity, thus revealing its classification as digital gold (Engle 2002).

\section{Results}

BTC-USD and GBTC: Unusual spikes in volume provide evidence of large institutional buying, especially when the pandemic hit, proving investor trust in Bitcoin as a hedge, as well as expected growth response to the halving. There is consistent holding support after every dip, and the volume is coherent with the price increase. A lot of sellers are present as well, which indicates trade ability, thus potential for currency value and a MoE. The volume is overall bullish, and significantly more traded than GLD or VOO, at a much lower market cap, which furthermore increases its stance as a SoV and MoE. GLD has a lot less volume and high price, compared to BTC-USD, which provides evidence that a small number of large investors still hold a belief in gold, and we can potentially interpret that they support ETFs (Exchange Traded Funds) in general. Conversely, the GBTC ETF has seen inflows, most probably from investors through traditional brokers. After every large selling, the volume does not seem to be persistent with the stock price, but decreases, thus signifying less trust. As to be expected, gold and Bitcoin behave similarly. Gold still lags behind Bitcoin in total return, though perhaps this could be due to market cap difference. Larger overall outflows are present within GLD, while BTC-USD has a lot more consistent purchasing volume. It is important to note that one of the factors helping the price increase of gold is also the inflation of the USD, therefore less overall value was added. VOO's volume is a lot lower than GLD, BTC-USD and GBTC, as well as return as an investment vehicle compared to BTC-USD.

\subsection{Volume Results}

The volume is also consistent with the price, although trading only occurs based on underlying economic performance as shown in the volume results in Figures 1-4.

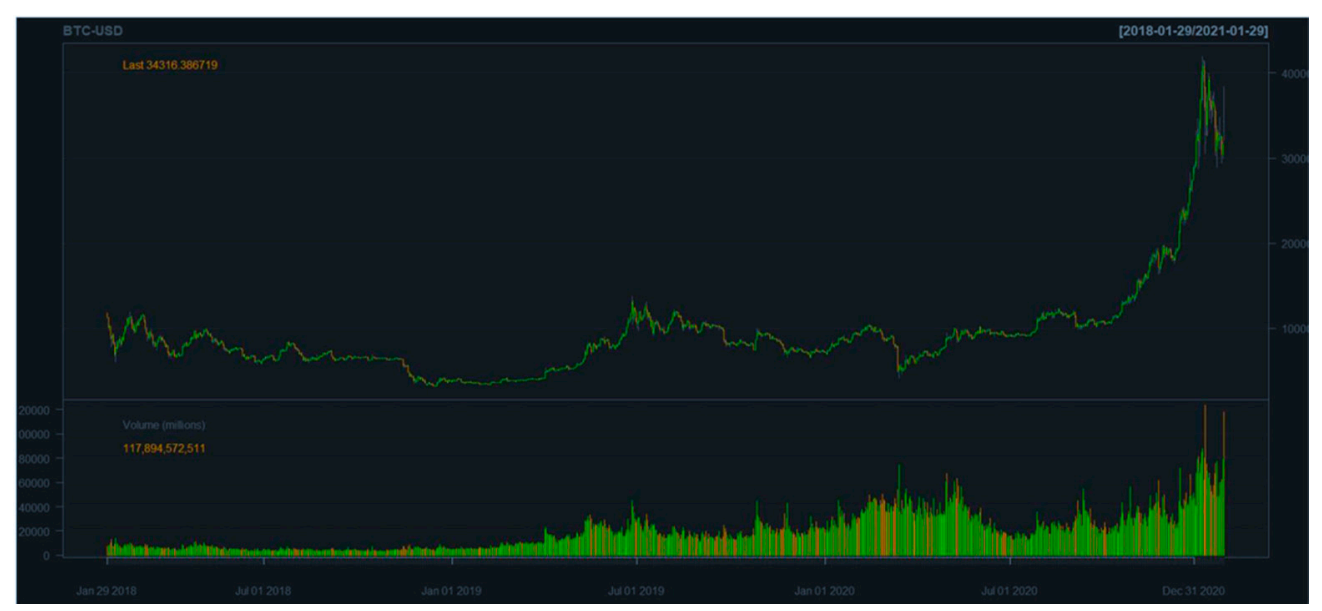

Figure 1. BTC-USD (Green: Buying Orders; Red: Selling Orders). 


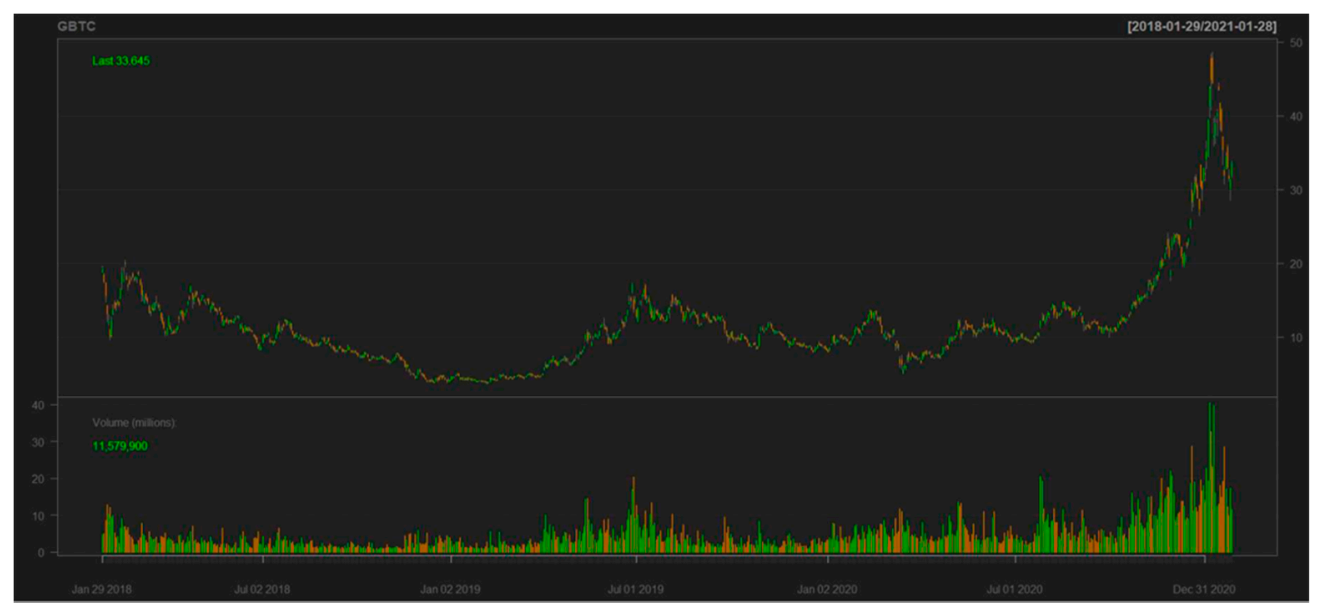

Figure 2. GBTC BTC-USD (Green: Buying Orders; Red: Selling Orders).

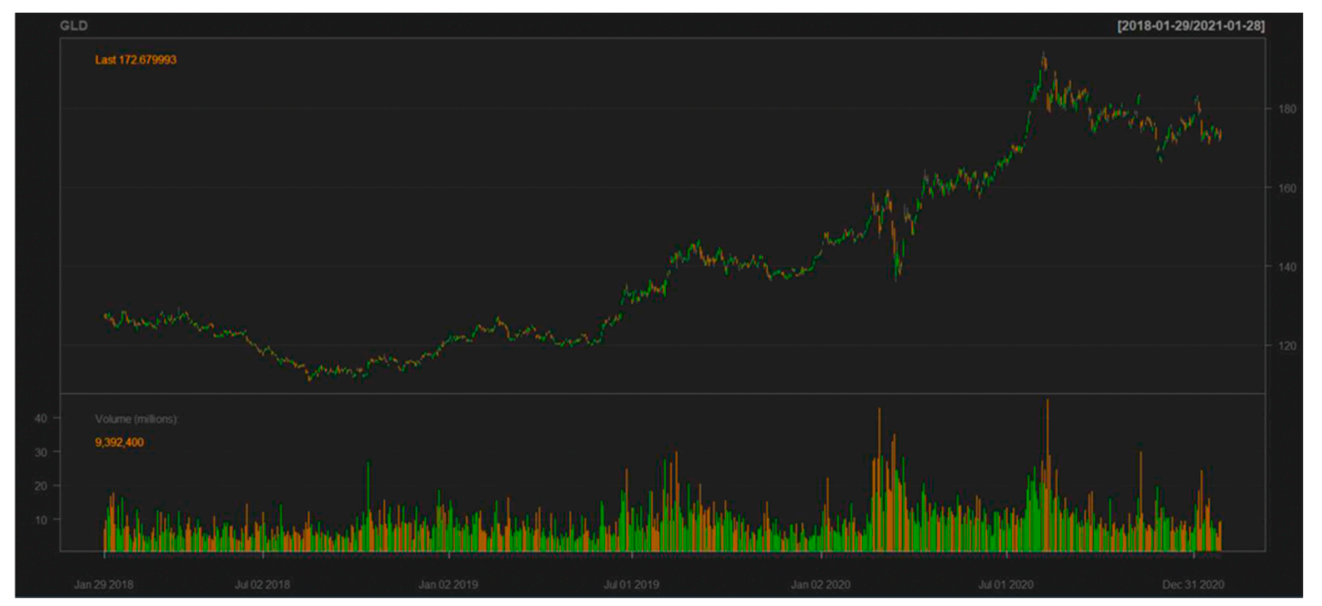

Figure 3. GLD BTC-USD (Green: Buying Orders; Red: Selling Orders).

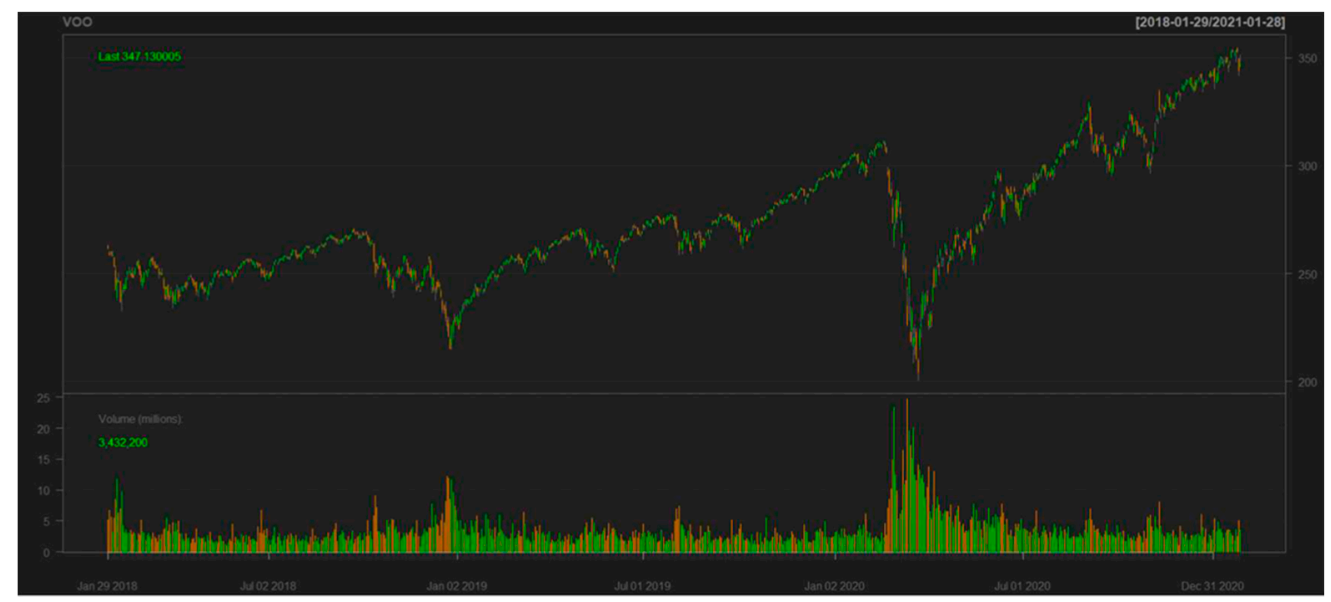

Figure 4. VOO BTC-USD (Green: Buying Orders; Red: Selling Orders).

\subsection{DCC-GARCH Model}

AR1 = coefficient of the mean model.

alpha1 $=$ coefficient to the squared residuals.

beta1 $=$ coefficient to the lagged variance. 


\subsection{Interpretation}

As shown in Tables 1 and 2, the autoregressive mean is negative in all three assets, meaning that the past values of the series will not predict the future value. The authors believe this to be an accurate assessment as models cannot model Black Swan events (i.e., the 2020 pandemic). Moreover, the 'beta1' coefficients are high in all three assets, indicating that past volatility shocks do affect conditional volatility which explains why volatility is clustered in Figures 3 and 5. The 'dcca1' value is insignificantly different from zero/approximately zero, and the 'bccb1' value is insignificantly different from 1 , but significantly different from 0 (Figures 6 and 7). As shown in Table 3 This indicates that the conditional correlation was constant during the experiment.

Table 1. Dynamic conditional correlation generalized autoregressive conditional heteroskedasticity (DCC GARCH) FIT.

\begin{tabular}{cc}
\hline Variable & Coefficient (Standard Error) \\
\hline Distribution and Model & $\begin{array}{c}\text { Mvnorm (Multi Variate Normal Distribution) and } \\
\text { DCC (Dynamic Conditional Correlation) (1.1) }\end{array}$ \\
\hline No. of parameters & 20 \\
\hline $\begin{array}{c}\text { [VAR (Vector Auto Regression) GARCH DCC } \\
\text { (Dynamic Conditional Correlation) UncQ] }\end{array}$ & {$[0+15+2+3]$} \\
\hline No. of Series & 356 \\
\hline No. of Observations & 6128.491 \\
\hline Log likelihood & 8.11 \\
\hline Av. Log likelihood & Information Criteria \\
\hline Akaike & -16.160 \\
\hline Bayes & -16.038 \\
\hline Shibata & -16.161 \\
\hline Hannah Quinn & -16.113 \\
\hline
\end{tabular}

Table 2. DCC multivariate GARCH (MGARCH) model optimal parameters.

\begin{tabular}{cccc}
\hline Variable & $\begin{array}{c}\text { GBTC (Grayscale } \\
\text { Bitcoin Trust Fund) }\end{array}$ & $\begin{array}{c}\text { VOO (Vanguard } \\
\text { 500 Index Fund) }\end{array}$ & GLD (Gold) \\
\hline $\mathrm{Mu}$ (mean) & 0.002025 & 0.001096 & 0.000135 \\
\hline $\begin{array}{c}\text { AR1 (coefficient of the mean } \\
\text { model) }\end{array}$ & -0.020731 & -0.069730 & -0.019810 \\
\hline Omega & 0.000345 & 0.000004 & 0.000004 \\
\hline $\begin{array}{c}\text { Alpha1 (coefficient to the } \\
\text { squared residuals) }\end{array}$ & 0.119798 & 0.235647 & 0.105659 \\
\hline $\begin{array}{c}\text { Beta1 (coefficient to the } \\
\text { lagged variance) }\end{array}$ & 0.775774 & 0.756135 & 0.859825 \\
\hline Dcca1 & 0.028476 & 0.028476 & 0.028476 \\
\hline Dccb1 & 0.951253 & 0.951253 & 0.951253 \\
\hline
\end{tabular}


Table 3. Correlation matrix of the returns on the last day.

\begin{tabular}{cccc}
\hline Tickers & GBTC & VOO & GLD \\
\hline GBTC & 1.00000000 & 0.07248292 & 0.11428288 \\
\hline VOO & 0.07248292 & 1.00000000 & 0.08309941 \\
\hline GLD & 0.11428288 & 0.08309941 & 1.00000000 \\
\hline
\end{tabular}
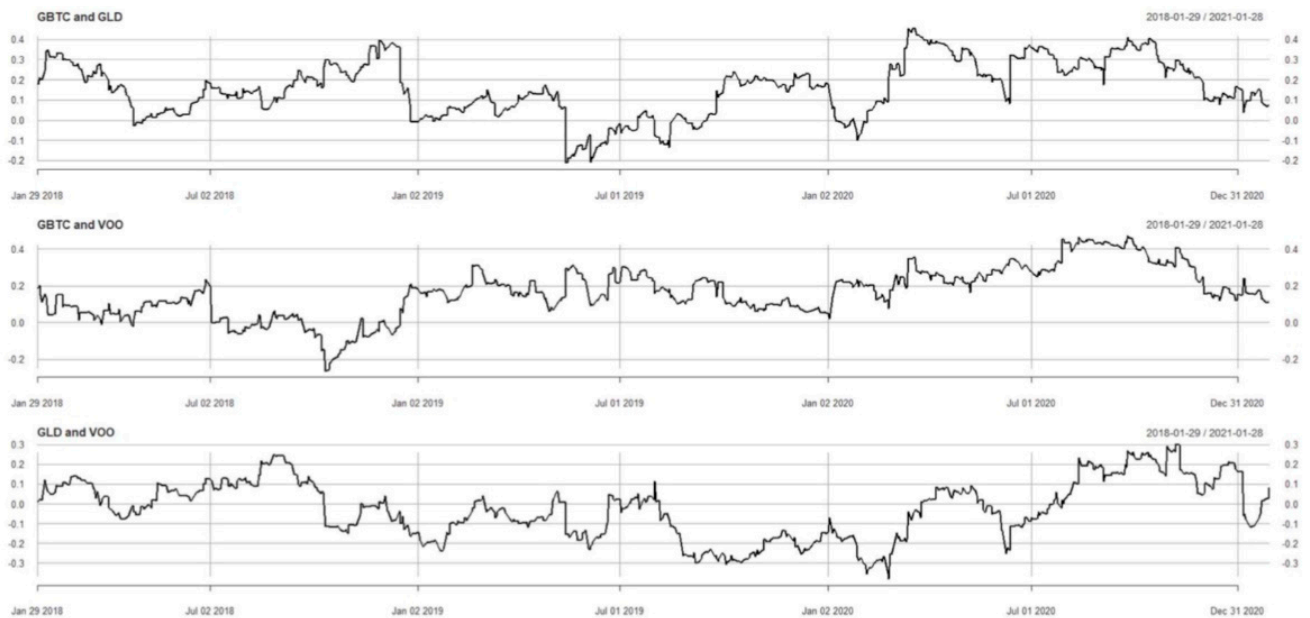

Figure 5. Correlation between GBTC, GLD and VOO.
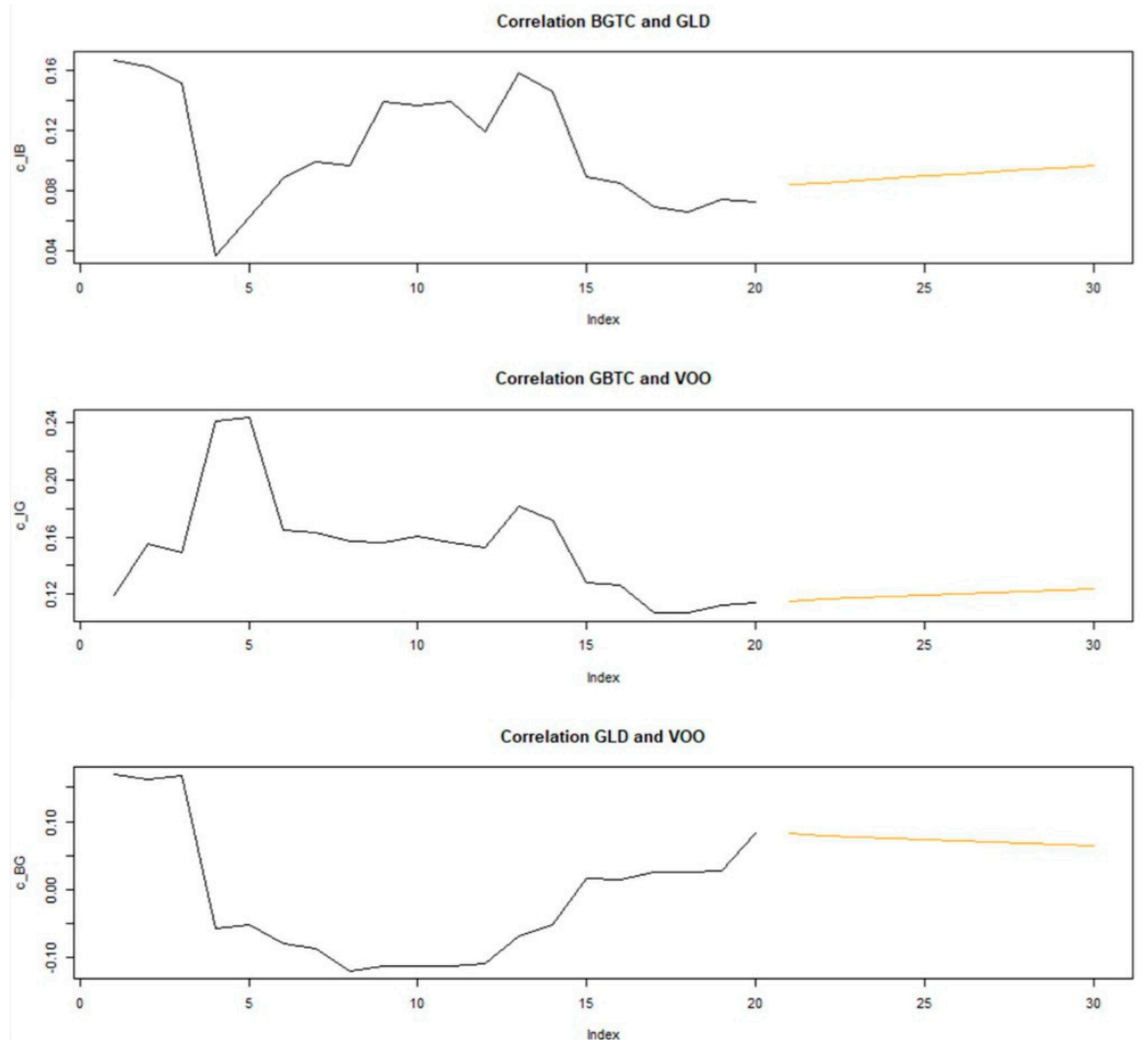

Figure 6. Correlation (Black) and forecast (yellow) between GLD, GBTC, and VOO. 


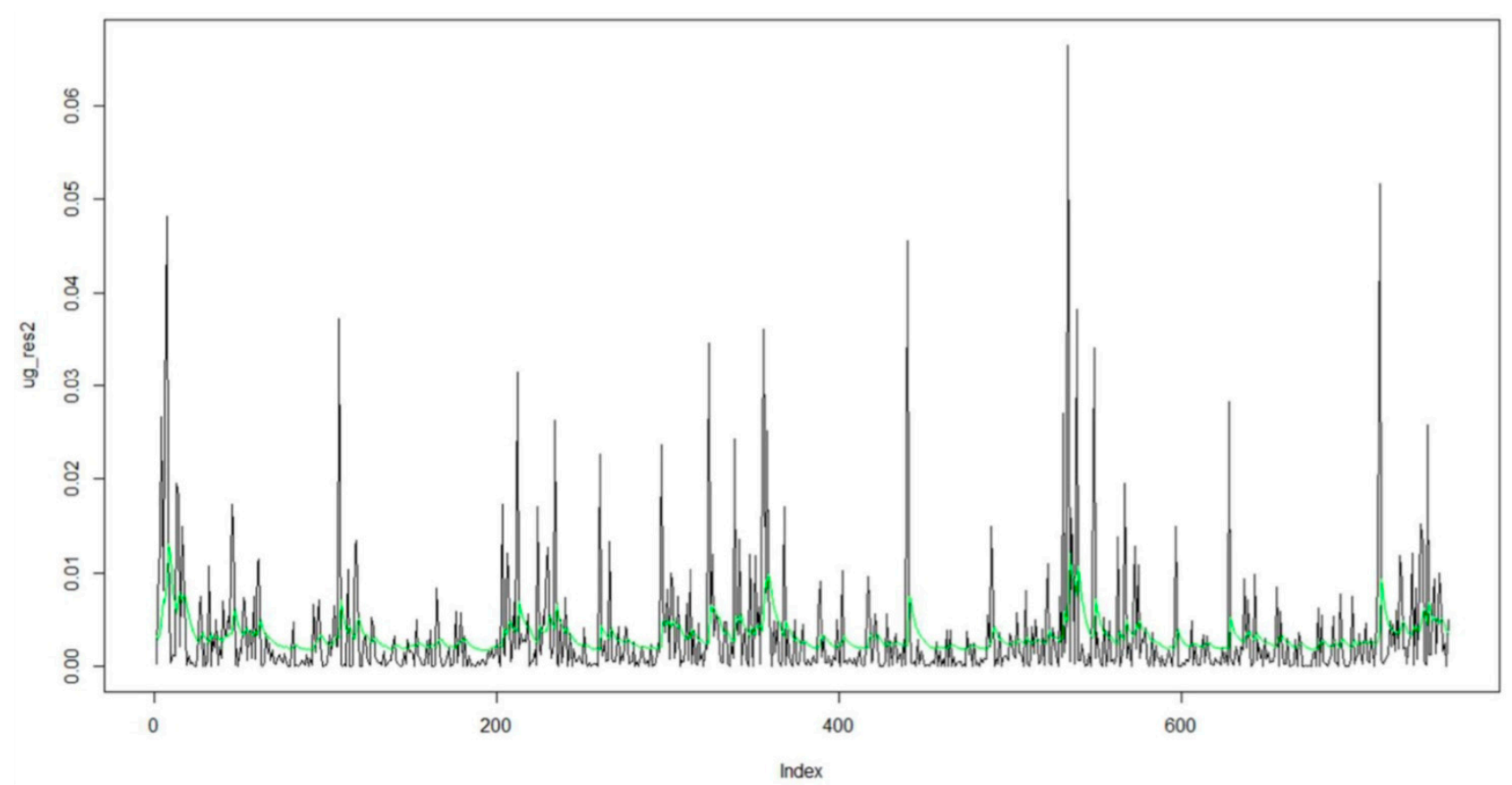

Figure 7. GBTC squared residuals (Black) and estimated conditional variance (Green).

\subsection{Correlation Interpretation}

The correlation between GBTC and GLD is positively low as the COVID-19 pandemic broke, indicating investors perceived Bitcoin as a digital hedge storage against the recession. Similarly, a comparison between GBTC and VOO indicates that investors perceived Bitcoin as an investment vehicle most of the time, except between July 2018 and January 2019. The GLD-VOO pair correlation fluctuation pre-February 2020 indicates that investors did not have as much confidence in gold as in Bitcoin for an investment vehicle, but still as a hedge during the pandemic. Figures 5-9 describe Bitcoin as the better hedge and investment between the three assets.

Black $=$ Last realised correlations.

Orange $=$ Forecasted correlation .

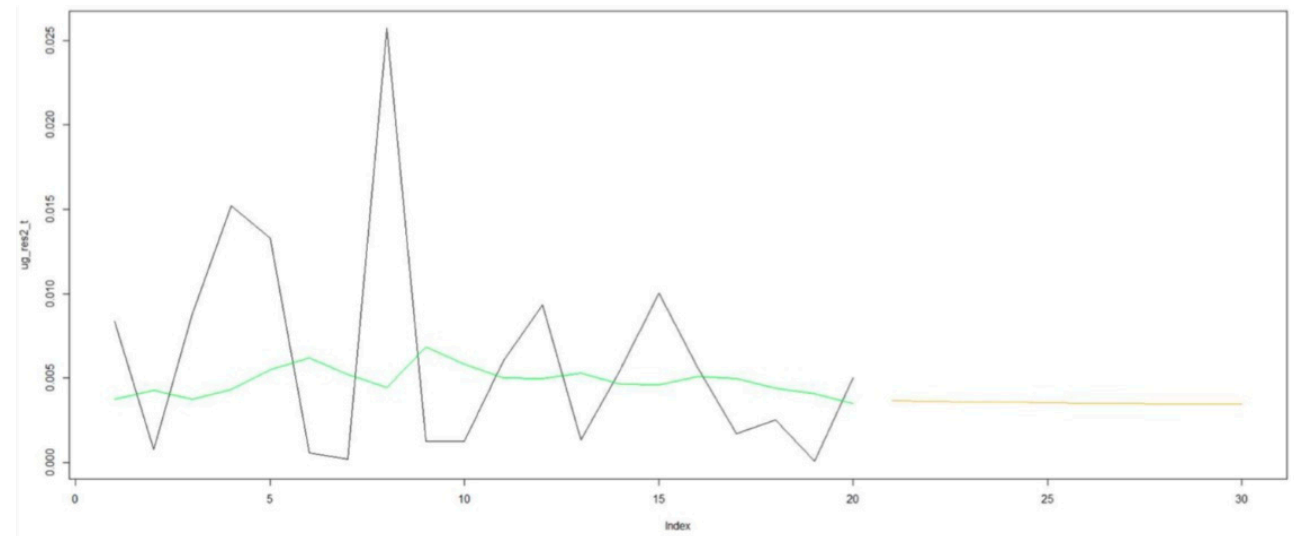

Figure 8. GBTC squared residuals (Black) of the last 20 observations, forecasted conditional variance (Yellow), estimated conditional variance (Green). 


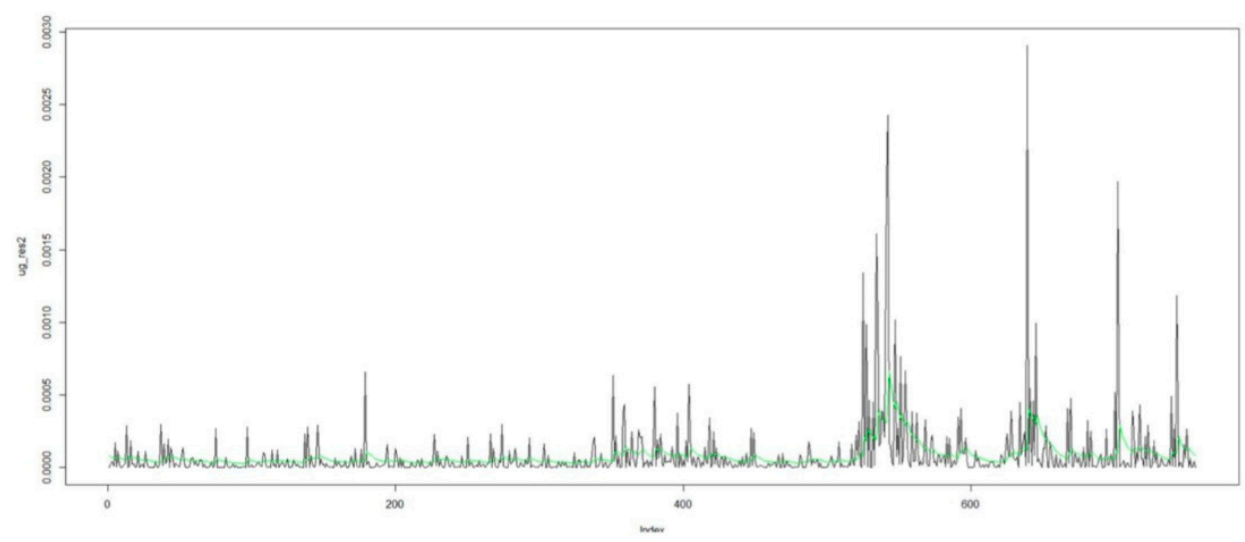

Figure 9. GLD squared residuals (Green) and estimated conditional variance (Green).

\subsection{Individual Assets}

Green $=$ Last estimated conditional variance.

Orange $=$ Forecast of conditional variance.

Black = Squared residuals of the last 20 observations.

Sigma Volatility forecasts (10 periods).

\subsection{Interpretation of Individual Figures}

All assets indicate volatility clustering. GBTC does not graph typical GARCH patterns. The residuals fluctuate, and estimated conditional variance only follows the squared residual above a certain threshold. The last estimated conditional variance decreases. The forecast of conditional variance picks up from the estimated conditional variance and continues towards unconditional variance value. Figure 5 indicates that GBTC and GLD are highly correlated as a hedge, meaning that investors are starting to adopt and hold BTC as digital gold, thus lower volatility was observed.

GLD has higher implied volatility than Bitcoin confirmed by Figure 10 which describes GLD behaving similarly to Bitcoin in terms of residual returns, and forecasted conditional volatility, although the estimated variance is not as coherent with residual returns as it is with GBTC.

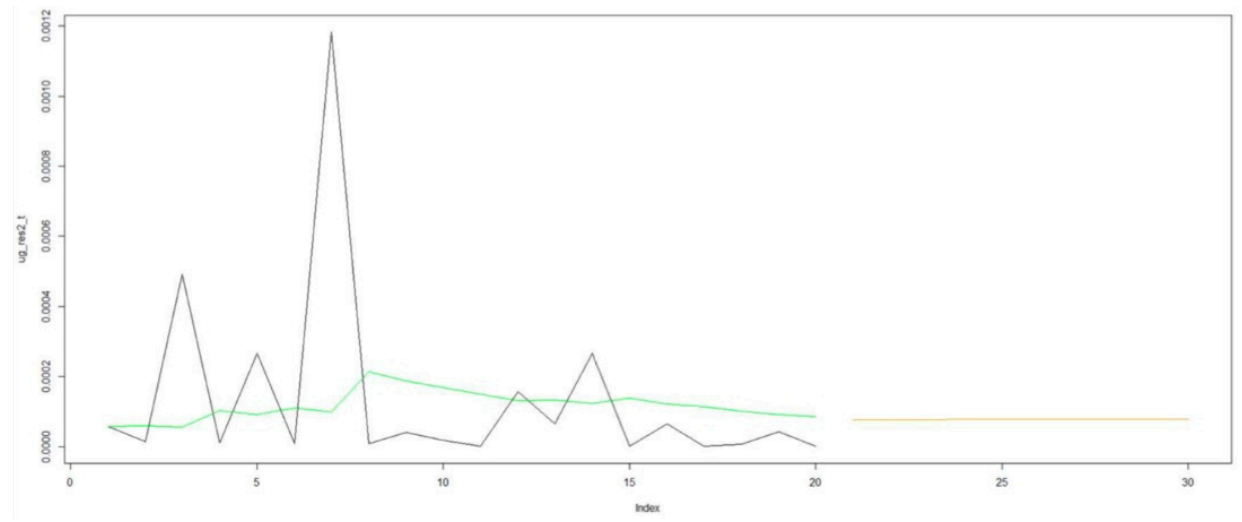

Figure 10. GLD squared residuals (Black) of the last 20 observations, forecasted (Yellow) conditional variance, estimated conditional variance (Green).

As expected, with VOO the estimated conditional variance adjusts with the squared residual which is not the case with GBTC and GLD (Figure 11). Unlike GBTC and GLD, VOO forecasts higher conditional variance in Figure 12. 


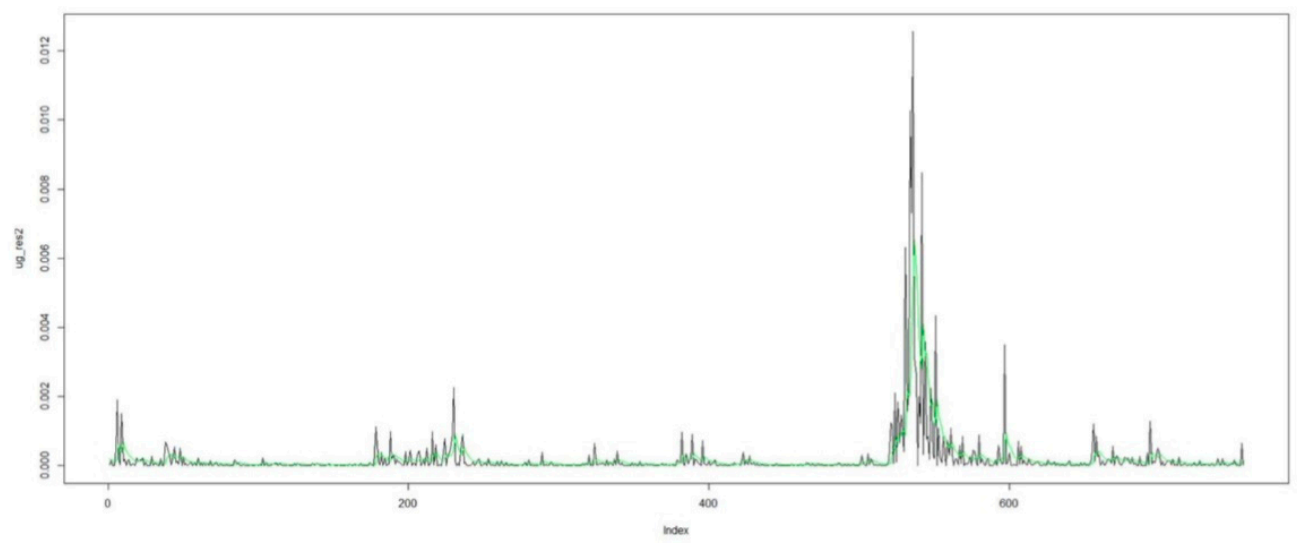

Figure 11. Squared residuals (Black) and estimated conditional variance (Green).

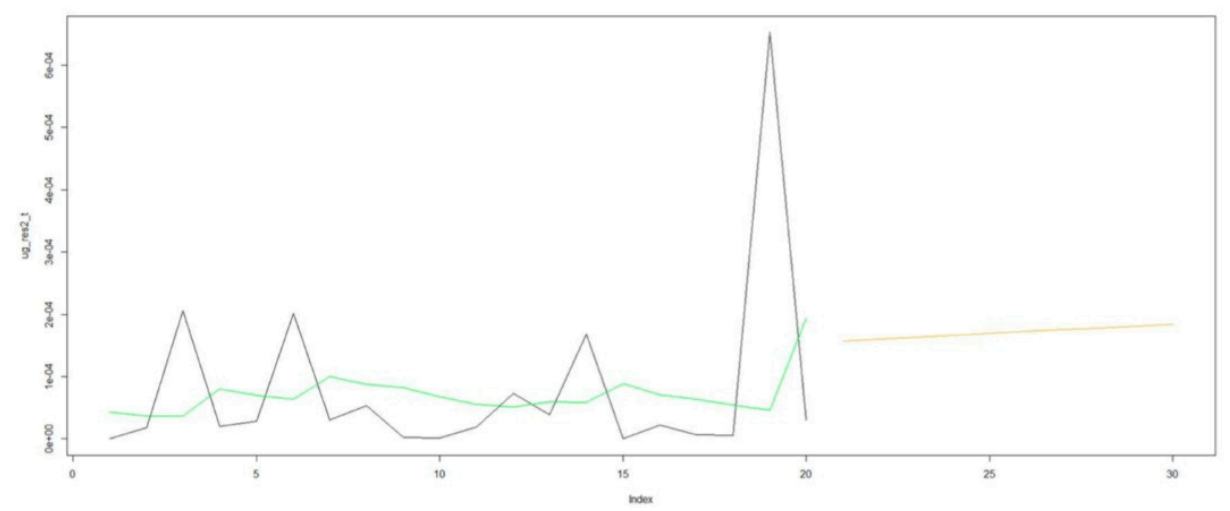

Figure 12. VOO squared residuals (Black) of the last 20 observations, forecasted (Yellow) conditional variance, estimated conditional variance (Green).

Additionally, the fluctuation of squared residuals is the opposite of both GLD and GBTC, described in Figure 7.

\section{Summary of Results}

A lot of research in cryptocurrencies has continued to explore whether Bitcoin could be considered a virtual gold (Klein et al. 2018) and its hedging ability in comparison to traditional assets (Wang et al. 2019). Bitcoin behaves very similarly to gold, but over time it has become less volatile with higher returns than gold and the major stock indexes (Jareño et al. 2020). This can be explained by halvings, regulation, adoption and investor perception as can be seen throughout this paper considering Bitcoin as a big bang alternative currency about to change the world (Kelly 2014). The results of this paper are in alignment with Dyhrberg (2016b) whose research show that bitcoin can clearly be used as a hedge against stocks in the Financial Times Stock Exchange Index and the American dollar in the short-term. Bitcoin thereby possess some of the same hedging abilities and safe-haven characteristics against specific uncertainties (Mokni et al. 2021). Like gold, Bitcoin can be included in the variety of tools available to market analysts to hedge market specific risks (Corbet et al. 2019), exhibiting a superiority over both gold and commodities (Bouri et al. 2020) and can be considered a major asset for portfolio diversification (Hatemi-J. et al. 2019).

\section{Conclusions, Limitations and Recommendations}

Since Bitcoin first launched in 2008, it has stood the test of time and persevered with resilience through various challenges. Every currency in history was eventually eroded, however Bitcoin stands to have the potential to be the first with no such cessation. It remains difficult to examine if the 2020 halving was priced in the increase of price due 
to volatility. Economically this is hard to do as one needs to deal with volatility risk. For new investors to benefit from a price increase from the halving supply reductions in the future, they have to consider necessary price volatility risk as Bitcoin becomes adopted over time (Hayek 1976). Since 2017, investors have shown trust towards Bitcoin becoming a medium of exchange and store of value, which will help it be considered a currency and a unit of account by 2140 . This can be contributed to by various underlying factors such as storage (wallets), regulations (exchanges), soft fork improvements, publicly traded company adoption of BTC, halvings, an overall more technologically inclined and digitally informed generation. Bitcoin now has the ability to compete with gold as an alternative hedge, as an investment with standard asset classes, a store of value as well as the cyberspace reserve currency. We can conclude that the interpretations raise the awareness of investors and policy makers about the importance of Bitcoin (Zhao and Zhang 2021) as a MoE, and SoV which can be described in figures of volume, correlation, residual returns and variances. Although volatility clustering is naturally present, volatility has decreased over time, giving Bitcoin a strong and justified support to become accepted as the new digital gold.

The limitations of this paper are that there is not much recent scholarly work and academic research. Bitcoin is still an ongoing experiment (Cermak 2017). Measures such as hacks and governmental involvement in the internet are not included, although that would be hard to imagine as the whole financial system is already tied to the internet (the world's central nervous system). Other limitations include the model itself as it has not been tested for skewness and kurtosis (Andersen and Bollerslev 1988) as well as Bitcoin's prophecy relying on market sentiment and the nature of human wants, as opposed to standard financial analysis. Although the GBTC is pegged onto the BTC-USD, the model could have used the BTC-USD pair as an even more accurate assessment if the data were available at the time of conducting the experiment and analysis. The selling of Satoshi's coins or identification of identity as the "founder" of Bitcoin could also be identified as a limitation or a gap. If this would occur, the Bitcoin thesis would not hold (Wray 2015). One can infer about the skill set of the person such as: capability of network programming, a good grasp of economic game theory understanding, a reasonable grasp of applied cryptography, and a system designer perspective to assemble something in a new way and most likely an expert programmer of remailer technology. One can suspect that 'Satoshi Nakamoto' is a single person due to consistency of code and the difficulty of keeping privacy and secrets across a group and, therefore, the said limitation is not a concern (Wray 2015). The secret identity helps Bitcoin fulfill its prophecy as humans tend to have a background in societal hierarchical thinking. Satoshi not acknowledging ownership has some important advantages, such as individuals not having anyone to reach out to ask or demand changes, thus all the focus is on Bitcoin itself. One needs to remember that legitimacy is one of the scarcest resources.

Recommendations for further research should be conducted in new cryptography as it is the building block of the digital world (Grinberg 2011) as well as considering adding a robustness analysis involving another method (e.g., ADCC; etc.) (Nugroho 2021) to test the robustness of the obtained results. Humanity needs to determine whether the current consensus is dependable in the long term (Leerssen 2021). Additionally, the ability of buying Bitcoin with cryptocurrencies that are fully anonymous needs discussion as they support illicit dealings (Philipson 2020). This is true as one can expect a rise of fully anonymous currencies over time. Lastly, more research is recommended on ethical considerations with decentralised technology, and its application of smart contracts (Alotaibi 2021).

As conditions of smart contracts are abstract, they can be used to implement logic and procedures in domains beyond commercial agreements such as universally accepted rules and models of governance (Rozas et al. 2021).

Satoshi solved a problem that humanity has never solved before: a governance problem of organising networks. The ability to tell stories and unite makes humans 
the most powerful creatures in the world. Stories allow us to create networks (e.g., money, the United States, communities). The fundamental question is the model of governance. Historically, answers have been a king or authority, an aristocracy, a commons which always leads to the tragedy of the commons. Nakamoto created a merit-based, democratically open network. Labour is the oldest and most fought over form of leverage to create wealth and change. Decentralisation is a fundamentally different way of leveraging humans with the ability to create trust between strangers without an entity or individual being in charge. The authors believe the research recommendations are a social duty as they will affect how humanity lives.

Author Contributions: All authors contributed equally to the conceptualization, methodology, software, validation, data analysis and execution, writing - review and editing, visualization, and supervision of this paper at all stages. All authors have read and agreed to the published version of the manuscript.

Funding: This research received funding from Geneva Business School.

Conflicts of Interest: The authors declare no conflict of interest.

\section{References}

Acharya, Sunayan, and Jessica Dunn. 2014. Overstock. com ventures into digital currencies. Journal of Business Cases and Applications 12: 1.

Amadeo, Kimberly, and J. Michael Boyle. 2020. US national debt by year compared to GDP and major events. The Balance 1: 1. Available online: https:/ / www.thebalance.com/national-debt-by-year-compared-to-gdp-and-major-events-3306287 (accessed on 10 August 2021).

Andersen, Torben G., and Tim Bollerslev. 1988. Answering the skeptics: Yes, standard volatility models do provide accurate forecasts. International Economic Review 39: 885-905. [CrossRef]

Andersson-Säll, Tim, and Johan Lindskog. 2019. A Study on the DCC Garch Model's Forecasting Ability with value at risk applications on the Scandinavian foreign exchange market. Available online: http://urn.kb.se/resolve?urn=urn:nbn:se:uu:diva-375201 (accessed on 8 July 2021).

Antonakakis, Nikolaos, and Julia Darby. 2013. Forecasting volatility in developing countries' nominal exchange returns. Applied Financial Economics 23: 1675-91. [CrossRef]

Alotaibi, Sara Jeza. 2021. Using Blockchain for Smart Contracts. In Innovative and Agile Contracting for Digital Transformation and Industry IGI Global 4: 208-221.

Bauwens, Luc, Sébastien Laurent, and Jeroen VK Rombouts. 2006. Multivariate GARCH models: A survey. Journal of Applied Econometrics 21: 79-109. [CrossRef]

Bianchi, Daniele, Luca Rossini, and Matteo Iacopini. 2020. Stablecoins and cryptocurrency returns: Evidence from large bayesian vars. SSRN. [CrossRef]

Bohte, Rick, and Luca Rossini. 2019. Comparing the forecasting of cryptocurrencies by bayesian time-varying volatility models. Journal of Risk and Financial Management 12: 150. [CrossRef]

Borradaile, Glencora. 2021. Defend Dissent. Corvallis: Oregon State University.

Bouri, Elie, Peter Molnár, Georges Azzi, David Roubaud, and Lars Ivar Hagfors. 2017. On the hedge and safe haven properties of Bitcoin: Is it really more than a diversifier? Finance Research Letters 20: 192-98. [CrossRef]

Bouri, Elie, Syed Jawad Hussain Shahzad, David Roubaud, Ladislav Kristoufek, and Brian Lucey. 2020. Bitcoin, gold, and commodities as safe havens for stocks: New insight through wavelet analysis. The Quarterly Review of Economics and Finance 77: 156-64. [CrossRef]

Boyapati, Vijay. 2018. The Bullish Case for Bitcoin. Available online: https://vijayboyapati.medium.com/the-bullish-case-for-bitcoin6 ecc8bdecc1 (accessed on 3 July 2021).

Breedlove, Robert. 2019. An Open Letter to Ray Dalio: Bitcoin. Available online: https://breedlove22.medium.com/an-open-letter-toray-dalio-re-bitcoin-4b07c52a1a98 (accessed on 7 July 2021).

Brownlees, Christian T., Robert F. Engle, and Bryan T. Kelly. 2011. A practical guide to volatility forecasting through calm and storm. SSRN. [CrossRef]

Catania, Leopoldo, Stefano Grassi, and Francesco Ravazzolo. 2019. Forecasting cryptocurrencies under model and parameter instability. International Journal of Forecasting 35: 485-501. [CrossRef]

Cermak, Vavrinec. 2017. Can bitcoin become a viable alternative to fiat currencies? An empirical analysis of bitcoin's volatility based on a GARCH model. In An Empirical Analysis of Bitcoin's Volatility Based on a GARCH Model. New York: Skidmore College.

Chen, Weili, Jun Wu, Zibin Zheng, Chuan Chen, and Yuren Zhou. 2019. Market manipulation of bitcoin: Evidence from mining the Mt. Gox transaction network. Paper presented at IEEE INFOCOM 2019-IEEE Conference on Computer Communications, Paris, France, April 29-May 2; pp. 964-72. 
Clegg, Alastair G. 2014. Could Bitcoin Be a Financial Solution for Developing Economies. Birmingham: University of Birmingham, vol. 1, pp. 2013-14.

Connors, Louisa, and William Mitchell. 2017. Framing modern monetary theory. Journal of Post Keynesian Economics 40: $239-59$. [CrossRef]

Corbet, Shaen, Brian Lucey, Andrew Urquhart, and Larisa Yarovaya. 2019. Cryptocurrencies as a financial asset: A systematic analysis. International Review of Financial Analysis 62: 182-99. [CrossRef]

Cuñado, Jorge Hernando, Jorge Colvin Díez, and Javier Antonio Enríquez Román. 2020. Engie: Business Model Transformation. Harvard Deusto Business Research 9: 152-67. [CrossRef]

Datta, Anwitaman, Sonja Buchegger, Le-Hung Vu, Thorsten Strufe, and Krzysztof Rzadca. 2010. Decentralized online social networks. In Handbook of Social Network Technologies and Applications. Boston: Springer, pp. 349-78.

Davidson, Laura, and Walter E. Block. 2015. Bitcoin, the Regression Theorem, and the emergence of a new medium of exchange. Quarterly Journal of Austrian Economics 18: 311.

De Grauwe, Paul. 1988. Exchange rate variability and the slowdown in growth of international trade. Staff Papers 35: 63-84. [CrossRef]

Dutta, Anupam, Debojyoti Das, R. K. Jana, and Xuan Vinh Vo. 2020. COVID-19 and oil market crash: Revisiting the safe haven property of gold and Bitcoin. Resources Policy 69: 101816. Available online: https://www.sciencedirect.com/science/article/abs/pii/S030 1420720308485 (accessed on 10 August 2021). [CrossRef]

Dyhrberg, Anne Haubo. 2016a. Bitcoin, gold and the dollar-A GARCH volatility analysis. Finance Research Letters 16: 85-92. [CrossRef] Dyhrberg, Anne Haubo. 2016b. Hedging capabilities of bitcoin. Is it the virtual gold? Finance Research Letters 16: 139-44. [CrossRef]

Elliott, Colin P. 2015. The Crisis of AD 33: Past and present. Journal of Ancient History 3: 267-81. [CrossRef]

Engel, Charles, and Kenneth D. West. 2005. Exchange rates and fundamentals. Journal of Political Economy 113: 485-517. [CrossRef]

Engle, Robert F. 1982. Autoregressive conditional heteroscedasticity with estimates of the variance of United Kingdom inflation. Econometrica: Journal of the Econometric Society, 987-1007. [CrossRef]

Engle, Robert. 2002. Dynamic conditional correlation: A simple class of multivariate generalized autoregressive conditional heteroskedasticity models. Journal of Business \& Economic Statistics 20: 339-50.

Frino, Alex, Steven Lecce, and Reuben Segara. 2011. The impact of trading halts on liquidity and price volatility: Evidence from the Australian Stock Exchange. Pacific-Basin Finance Journal 19: 298-307. [CrossRef]

Fröhlich, Michael, Maurizio Raphael Wagenhaus, Albrecht Schmidt, and Florian Alt. 2021. Don't Stop Me Now! Exploring Challenges of First-Time Cryptocurrency Users. Paper presented at Designing Interactive Systems Conference, Online, June 21-July 1; pp. $138-48$.

Fukami, Aya, Radina Stoykova, and Zeno Geradts. 2021. A new model for forensic data extraction from encrypted mobile devices. Forensic Science International: Digital Investigation 38: 301169.

Garman, Mark B., and Michael J. Klass. 1980. On the estimation of security price volatilities from historical data. Journal of business 53: 67-78. [CrossRef]

Ghalanos, Alexios. 2019. The RMGARCH Models: Background and Properties. Version 1.3-0. Available online: https://mran. microsoft.com (accessed on 19 June 2021).

Griffin, John M., and Amin Shams. 2020. Is Bitcoin really untethered? The Journal of Finance 75: 1913-64. [CrossRef]

Grinberg, Reuben. 2011. Bitcoin: An innovative alternative digital currency. Hastings Science E Technology Law Journal 4: 160.

Gronwald, Marc. 2014. The Economics of Bitcoins-Market Characteristics and Price Jumps. Available online: https://ssrn.com/ abstract=2548999 (accessed on 2 June 2021).

Hansen, Peter R., and Asger Lunde. 2005. A forecast comparison of volatility models: Does anything beat a GARCH (1, 1)? Journal of Applied Econometrics 20: 873-89. [CrossRef]

Hatemi-J., Abdulnasser, Mohamed A. Hajji, Elie Bouri, and Rangan Gupta. 2019. The Benefits of Diversification between Bitcoin, Bonds, Equities and the US Dollar: A Matter of Portfolio Construction. No. 201959. Pretoria: University of Pretoria.

Hayek, Friedrich August. 1976. Choice in Currency: A Way to Stop Inflation. Auburn: Ludwig von Mises Institute.

Hossain, Md Jamal, Mohd Tahir Ismail, Sadia Akter, and Mohammad Raquibul Hossain. 2020. Which will serve better as a hedge or diversifier Gold or Bitcoin? Paper presented at 2020 International Conference on Decision Aid Sciences and Application (DASA), Online, November 8-9; pp. 1052-55.

Jareño, Francisco, María de la O González, Marta Tolentino, and Karen Sierra. 2020. Bitcoin and gold price returns: A quantile regression and NARDL analysis. Resources Policy 67: 101666. [CrossRef]

Jiang, Yonghong, Jiayi Lie, Jieru Wang, and Jinqi Mu. 2021. Revisiting the roles of cryptocurrencies in stock markets: A quantile coherency perspective. Economic Modelling 95: 21-34. [CrossRef]

Jones, Paul, and Lorenzo Giorgianni. 2020. The Great Monetary Inflation. Market Outlook-Macro Perspective. Available online: coinvertit.com (accessed on 15 May 2021).

Kelly, Brian. 2014. The Bitcoin Big Bang: How Alternative Currencies Are about to Change the World. Hoboken: John Wiley \& Sons.

Kim, Jung. 2021. Most Traded National Currencies for Bitcoin. Coinhills. Available online: https://www.coinhills.com/market/ currency/ (accessed on 13 March 2021).

Klein, Tony, Hien Pham Thu, and Thomas Walther. 2018. Bitcoin is not the New Gold-A comparison of volatility, correlation, and portfolio performance. International Review of Financial Analysis 59: 105-16. [CrossRef] 
Kliber, Agata, Paweł Marszałek, Ida Musiałkowska, and Katarzyna Świerczyńska. 2019. Bitcoin: Safe haven, hedge or diversifier? Perception of bitcoin in the context of a country's economic situation-A stochastic volatility approach. Physica A: Statistical Mechanics and Its Applications 524: 246-57. [CrossRef]

Knafo, Samuel. 2006. The gold standard and the origins of the modern international monetary system. Review of International Political Economy 13: 78-102. [CrossRef]

Küfeoglu, Sinan, and Mahmut Özkuran. 2019. Energy Consumption of Bitcoin Mining. Available online: repository.cam.ac.uk (accessed on 2 July 2021).

Kurtz, Marcus J., and Andrew Schrank. 2007. Growth and governance: Models, measures, and mechanisms. The Journal of Politics 69: 538-54. [CrossRef]

Lamport, Leslie, Robert Shostak, and Marshall Pease. 2019. The Byzantine generals problem. Concurrency: The Works of Leslie Lamport 1: 203-26.

Laurent, Sébastien, Jeroen V.K. Rombouts, and Francesco Violante. 2012. On the forecasting accuracy of multivariate GARCH models. Journal of Applied Econometrics 27: 934-55. [CrossRef]

Lavoie, Marc. 2014. Post-Keynesian Economics: New Foundations. Cheltenham: Edward Elgar Publishing.

Leerssen, Joep. 2021. Culture, humanities, evolution: The complexity of meaning-making over time. Philosophical Transactions of the Royal Society B 376: 20200043. [CrossRef]

Luther, William J., and Alexander W. Salter. 2017. Bitcoin and the bailout. The Quarterly Review of Economics and Finance 66: 50-56. [CrossRef]

Maddox, Alexia, and Luke J. Heemsbergen. 2021. Digging in Crypto-Communities' Future-Making: From Dark to Doge. M/C Journal 24. [CrossRef]

Masters, Jered. 2019. Impact of the 2020 Bitcoin Halving: A Mathematical, Social, and Econometric Analysis, Bitcoin 2020 Having Impact Analysis. Bentley: Curtin University.

Mokni, Khaled, Elie Bouri, Ahdi Noomen Ajmi, and Xuan Vinh Vo. 2021. Does Bitcoin Hedge Categorical Economic Uncertainty? A Quantile Analysis. Thousand Oaks: SAGE Open.

Nabilou, Hossein. 2020. Testing the waters of the Rubicon: The European Central Bank and central bank digital currencies. Journal of Banking Regulation 21: 299-314. [CrossRef]

Nakamoto, Satoshi. 2009. Bitcoin: A peer-to-peer electronic cash system Bitcoin: A Peer-to-Peer Electronic Cash System. Bitcoin. org. Available online: https:/ / bitcoin.org/en/bitcoin-paper (accessed on 19 March 2021).

Nugroho, Bayu Adi. 2021. Dynamic risk-based optimization on cryptocurrencies. Journal of Capital Markets Studies. [CrossRef]

Orskaug, Elisabeth. 2009. Multivariate DCC Garch Model: With Various Error Distributions. Master's Dissertation, Aalborg University, Aalborg, Denmark.

Peterson, Timothy. 2018. Metcalfe's Law as a Model for Bitcoin's Value. Alternative Investment Analyst Review Q 7: 9-18. [CrossRef]

Philipson, Sarah. 2020. Consumers and enterprises as actors on the market. Harvard Deusto Business Research 9: 168-80. [CrossRef]

Ponticelli, Jacopo, and Hans Joachim Voth. 2011. Austerity and Anarchy: Budget Cuts and Social Unrest in Europe, 1919-2008. Masters dissertation, Pompeu Fabra University, Barcelona, Spain; p. 10230.

Poon, Ser-Huang. 2005. A Practical Guide to Forecasting Financial Market Volatility. Hoboken: John Wiley \& Sons.

Putnam, Blu. 2020. The Fed's Balance Sheet Will Continue to Grow in 2021. Chicago: CME Group.

Regaieg, Rym, Wajdi Moussa, and Nidhal Mgadmi. 2020. Can We Reckon Bitcoin as a Hedge, a Safe Haven or a Diversifier for US Dollars? Global Business Review. [CrossRef]

Rosales, Antulio. 2021. Unveiling the power behind cryptocurrency mining in Venezuela: A fragile energy infrastructure and precarious labor. Energy Research \& Social Science 79: 102167.

Rozas, David, Antonio Tenorio-Fornés, Silvia Díaz-Molina, and Samer Hassan. 2021. When Ostrom Meets Blockchain: Exploring the Potentials of Blockchain for Commons Governance. Thousand Oaks: SAGE Open.

Scott, Brett, John Loonam, and Vikas Kumar. 2017. Exploring the rise of blockchain technology: Towards distributed collaborative organizations. Strategic Change 26: 423-28. [CrossRef]

Selmi, Refk, Walid Mensi, Shawkat Hammoudeh, and Jamal Bouoiyour. 2018. Is Bitcoin a hedge, a safe haven or a diversifier for oil price movements? A comparison with gold. Energy Economics 74: 787-801. [CrossRef]

Senge, Peter M., Bryan Smith, Nina Kruschwitz, Joe Laur, and Sara Schley. 2008. The Necessary Revolution: How Individuals and Organizations Are Working together to Create a Sustainable World. Redfern: Currency.

Shahzad, Syed Jawad Hussain, Elie Bouri, David Roubaud, Ladislav Kristoufek, and Brian Lucey. 2019. Is Bitcoin a better safe-haven investment than gold and commodities? International Review of Financial Analysis 63: 322-30. [CrossRef]

Smales, Lee A. 2019. Bitcoin as a safe haven: Is it even worth considering? Finance Research Letters 30: 385-93. [CrossRef]

Stensås, Anders, Magnus Frostholm Nygaard, Khine Kyaw, and Sirimon Treepongkaruna. 2019. Can Bitcoin be a diversifier, hedge or safe haven tool? Cogent Economics \& Finance 7: 1593072.

Stevens, Amy, and James Allen-Robertson. 2021. Encrypting human rights: The intertwining of resistant voices in the UK state surveillance debate. Big Data E Society 8: 2053951720985304.

Stross, Randall. 2012. What's Coming Out of Silicon Valley. The New York Times, June 19.

Szabo, Nick. 2002. Shelling Out. Satoshi Nakamoto Institute. Available online: https://nakamotoinstitute.org/shelling-out (accessed on 23 February 2021). 
Takaishi, Tetsuya. 2020. Rough volatility of Bitcoin. Finance Research Letters 32: 101379. [CrossRef]

Takemoto, Yoshifumi, and Sophie Knight. 2014. Mt. Gox files for bankruptcy, hit with lawsuit. Reuters Accessed January 4: 2018.

Tsertsvadze, Vano, and Lali Khurtsia. 2015. Drugs, Silk Road, Bitcoins. WASET World Academy 17: 3612-15.

Umar, Muhammad, Chi-Wei Su, Syed Kumail Abbas Rizvi, and Xue-Feng Shao. 2021. Bitcoin: A safe haven asset and a winner amid political and economic uncertainties in the US? Technological Forecasting and Social Change 167: 120680. [CrossRef]

Wang, Gangjin, Yanping Tang, Chi Xie, and Shou Chen. 2019. Is bitcoin a safe haven or a hedging asset? Evidence from China. Journal of Management Science and Engineering 4: 173-88. [CrossRef]

Wang, Gang-Jin, Xin-yu Ma, and Hao-yu Wu. 2020. Are stablecoins truly diversifiers, hedges, or safe havens against traditional cryptocurrencies as their name sug-gests? Research in International Business and Finance 54: 101225. [CrossRef]

Wray, L. Randall. 2015. Modern Money Theory: A Primer on Macroeconomics for Sovereign Monetary Systems, 2nd ed. New York: Springer.

Yermack, David. 2015. Is Bitcoin a Real Currency? An Economic Appraisal. In Handbook of Digital Currency. Amsterdam: Academic Press, pp. 31-43.

Zhang, Yue-Jun, Elie Bouri, Rangan Gupta, and Shu-Jiao Ma. 2021. Risk spillover between Bitcoin and conventional financial markets: An expectile-based approach. The North American Journal of Economics and Finance 55: 101296. [CrossRef]

Zhao, Haidong, and Lini Zhang. 2021. Financial literacy or investment experience: Which is more influential in cryptocurrency investment? International Journal of Bank Marketing. [CrossRef] 